\title{
Correlation of Serum Testosterone Level and Morning Pulse in Athletes
}

\author{
Zhiyun Ma \\ School of Physical Education, Jiujiang University, Jiujiang, China \\ longhorse1971@163.com
}

\begin{abstract}
The present study aimed to investigate the relation between serum testosterone level and morning pulse of athletes during the summer training. The results showed values of serum testosterone and morning pulse were slightly changed on the beginning and end of the summer training, and the metaphase values of serum testosterone and morning pulse were both remarkably enhanced, compared with baseline. Furthermore, the serum testosterone level was markedly correlated with the morning pulse at four time points during the summer training above. In conclusion, there exists a significantly negative correlation between the serum testosterone level and the morning pulse in athletes during the summer training.

Index Terms - testosterone, morning pulse, correlation, athletes

\section{Introduction}

Serum testosterone level is one of the important indexes assessing the function condition of athletes. Morning pulse is frequently one of simply and practical indexes judging the fatigue degree of heart. Serum testosterone level and heart function are always dynamically changed under the condition of the exercise training. The present study hypothesizes that there is a certain correlation between Serum testosterone level and morning pulse in athletes.
\end{abstract}

\section{Materials and Methods}

\section{Subjects}

All 10 male swimming players (mean $\pm \mathrm{SD}$, age $18.6 \pm 1.9$ years, height $175 \pm 3.6 \mathrm{~cm}$, weight $72 \pm 4.7 \mathrm{~kg}$, training duration $8.7 \pm 2.1$ years) were healthy without the illness of incretion and cardiovascular system through the medical examination.

\section{Testosterone Measurement}

Players were at rest every Sunday. Venous blood sample was drawn from each player on an empty stomach at 7:00 8:00 on Monday of the first, second, fifth and tenth (last) week during the summer training, and used to detect the testosterone value of baseline, beginning, metaphase and end, respectively. The coagulated samples stationarily placed $1 \mathrm{~h}$ were centrifuged by $3500 \mathrm{rpm}$ for $8 \mathrm{~min}$, and the serum were taken to measure the testosterone levels. Radioimmunoassay (RIA) was utilized to determine the concentration of serum testosterone.

\section{Morning Pulse Measurement}

The heart rate of each player was measured three times post-wakeup in the early morning, and three results were averaged to acquire the value of the morning pulse. Four values of the morning pulse were acquired at the same four time points as testosterone measurement.

\section{Statistical Analysis}

SPSS18.0 was utilized to statistically analyze the data, and they were characterized as Mean \pm SD. $P<0.05$ were considered to be statistically significant.

\section{Results}

All the results were showed in Table 1.

Table 1. Serum testosterone level, morning pulse and their correlation in athletes $(n=10)$

\begin{tabular}{|l|l|l|l|l|l|}
\hline \multicolumn{1}{|l|}{ index } & baseline value & value of beginning & value of metaphase & value of end \\
\hline \multicolumn{2}{l|}{ testosterone (ng/dl) } & $561.21 \pm 108.65$ & $537.35 \pm 97.43$ & $439.67 \pm 83.82 *$ & $595.63 \pm 115.72$ \\
\hline \multicolumn{2}{|l|}{ morning pulse (b/min) } & $50.5 \pm 2.3$ & $52.3 \pm 1.9$ & $56.7 \pm 2.8 *$ & $49.3 \pm 1.8$ \\
\hline \multirow{2}{*}{ correlation } & $\mathrm{r}$ & -0.81 & -0.79 & -0.86 & -0.82 \\
\cline { 2 - 6 } & $p$ & $<0.05$ & $<0.05$ & $<0.05$ & $<0.05$ \\
\hline
\end{tabular}

$* P<0.05$, compared with baseline value

\section{Discussion}

The certain concentration of blood testosterone can attenuate the myocardial ischemia-reperfusion injury, fibrosis, apoptosis or necrosis, and enhance the myocardial force of contraction [1-3]. Testosterone functions Physiologically via combining with androgen receptor (AR), and AR exists in most of atrium, ventricle, aorta and coronary artery. Testosterone affects a variety of cells in the myocardial tissue to regulate the function of heart in the pathway of genome or non-genome [4-6]. According to the results of the present study, testosterone value of beginning, compared with the 
baseline value, was changed without statistical significance, which suggested that the incretion conditions of athletes were nice; Metaphase value dramatically decreased, which suggested that the incretion conditions of athletes were poor. Perhaps that had something to do with over-load in the metaphase; The testosterone value of end slightly increased, which suggested that the incretion functions of athletes recovered, perhaps that correlated with load adjustment or adaptation of athletes to training. The morning pulse of athlete is considerably stable under the condition of the good function. The results showed that metaphase morning pulse value, relative to the baseline value, markedly increased, which suggested that the heart disfunction of athletes happened. Maybe that correlated with over-load or overfatigue. In the present study, it was manifested that the morning pulse significantly correlated with serum testosterone level by analyzing their correlative coefficient and significance. Taken together, the heart function of athletes is apparently regulated by the serum testosterone on the ground of exercise training, and the morning pulse is remarkably and negatively correlated with the serum testosterone level.

\section{Conclusions}

There exists a significantly negative correlation between the serum testosterone level and the morning pulse in athletes during the summer training, and the relevant mechanism is to be elucidated.

\section{References}

[1] C.M. Webb, and P. Collins, Testosterone and coronary artery disease in men, Maturitas, vol. 67, no. 1, 2010, pp. 15-19.

[2] G. Guder, B. Allolio, C.E. Angermann, and S. Stork, Androgen deficiency in heart failure, Curr Heart Fail Rep, vol. 8, no. 2, 2011, pp. 131-139.

[3] M.D. Schwarcz, and W.H. Frishman, Testosterone and coronary artery disease, Cardiol Rev, vol. 18, no. 5, 2010, pp. 251-257.

[4] M. Pierdominici, E. Ortona, F. Franconi, M. Caprio, E. Straface, and W. Malorni, Gender specific aspects of cell death in the cardiovascular system, Curr Pharm Des, vol. 17, no. 11, 2011, pp. 1046-1055.

[5] D. Africander, N. Verhoog, and J.P. Hapgood, Molecular mechanisms of steroid receptor-mediated actions by synthetic progestins used in HRT and contraception, Steroids, vol. 76, no. 7, 2011, pp. 636-652.

[6] S. Tsang, J. Liu, and T.M. Wong, Testosterone and cardioprotection against myocardial ischemia, Cardiovasc Hematol Disord Drug Targets, vol. 7, no. 2, 2007, pp. 119-125. 